Document downloaded from:

http://hdl.handle.net/10251/65715

This paper must be cited as:

Guerra, T.; Sala, A.; Tanaka, K. (2015). Fuzzy control turns 50: 10 years later. Fuzzy Sets and Systems. 281:162-182. doi:10.1016/j.fss.2015.05.005.

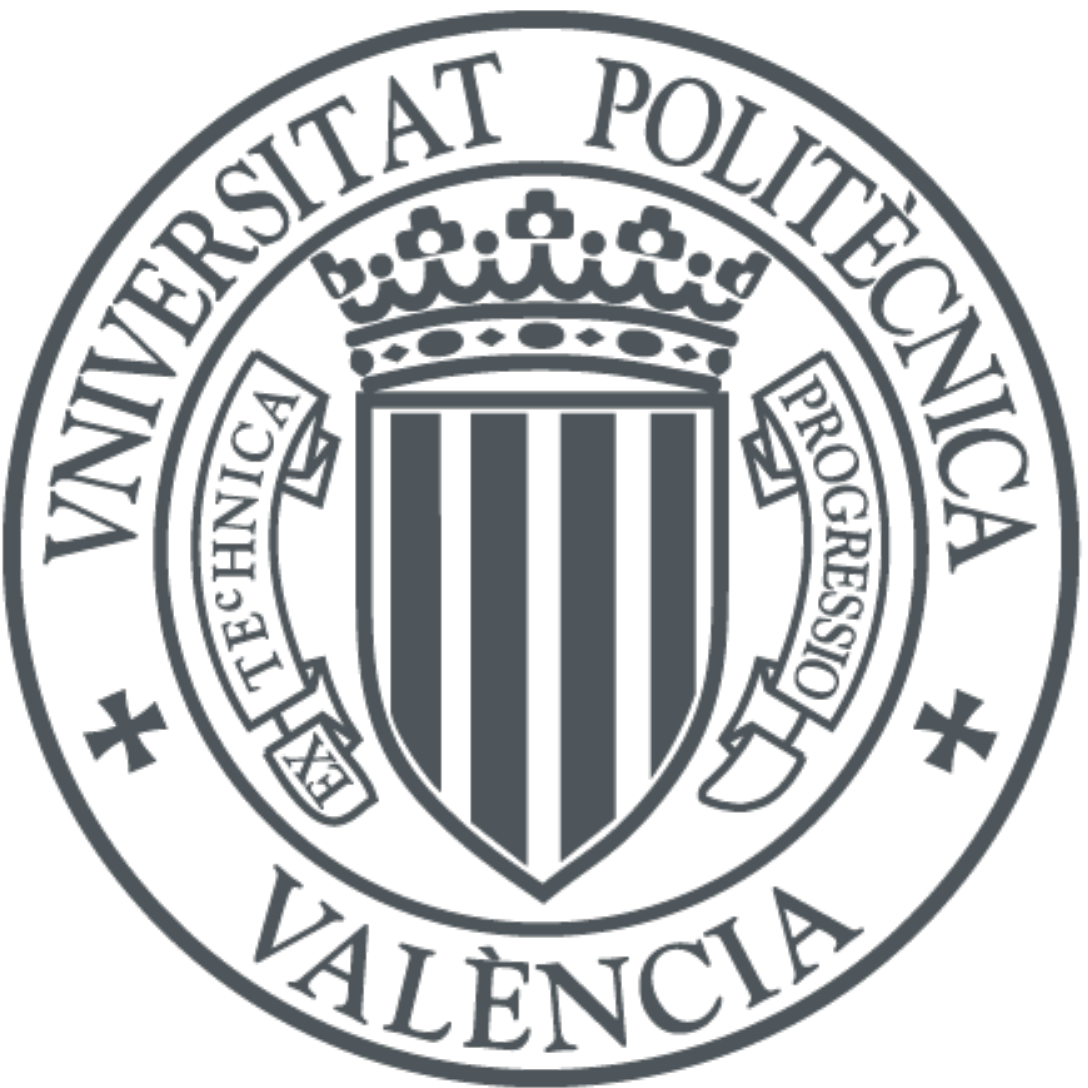

The final publication is available at

http://dx.doi.org/10.1016/j.fss.2015.05.005

Copyright Elsevier

Additional Information 


\title{
Fuzzy control turns 50: 10 years later
}

\author{
Thierry M. Guerra ${ }^{\mathrm{a}}$, Antonio Sala*b ${ }^{*}$ and Kazuo Tanaka ${ }^{\mathrm{c}}$
}

\begin{abstract}
${ }^{a}$ University of Valenciennes and Hainaut-Cambresis, LAMIH (UMR CNRS 8201), Le Mont Houy, 59313 Valenciennes Cedex 9, France ${ }^{b}$ Inst. Universitario de Automática e Informática Industrial, Universitat Politecnica de Valencia, Camino de Vera, S/N 46022 Valencia, Spain; *Corresponding author. Phone: (+34)963877000, email: asala@isa.upv.es

${ }^{c}$ Dept. Mech. Eng. and Intelligent Systems, The University of Electro-Communications, 1-5-1 Chofugaoka, Chofu, Tokyo 182-8585 Japan
\end{abstract}

\begin{abstract}
In 2015, we celebrate the 50th anniversary of Fuzzy Sets, ten years after the main milestones regarding its applications in fuzzy control in their 40th birthday were reviewed in FSS, see [1]. Ten years is at the same time a long period and short time thinking to the inner dynamics of research. This paper, presented for these 50 years of Fuzzy Sets is taking into account both thoughts. A first part presents a quick recap of the history of fuzzy control: from model-free design, based on human reasoning to quasi-LPV (Linear Parameter Varying) model-based control design via some milestones, and key applications. The second part shows where we arrived and what the improvements are since the milestone of the first 40 years. A last part is devoted to discussion and possible future research topics.
\end{abstract}

\section{The sound of the past: Fuzzy Control's first 40 years}

Fuzzy control is almost as old as fuzzy sets themselves. However, its popularity and acceptance have been (and, perhaps, still are) subject to fierce controversy. In order to recall to "young" readers what the atmosphere from the old times was we will pick up some sentences from fuzzy opponents:

"Fuzzification" is a kind of scientific permissiveness. It tends to result in socially appealing slogans unaccompanied by the discipline of hard scientific work and patient observation.

Prof. Rudolf Kalman Univ Florida, 1972 (cited in: [2])

Fuzziness is probability in disguise. I can design a controller with probability that could do the same thing that you could do with fuzzy logic.

Prof. Myron Tribus, UCLA (Bayesian) on hearing of the fuzzy-logic control of the Sendai subway system, may 1988 [3]

Mamdani type control systems are only appropriate for "toy class" problems.

Prof. Michael Athans, MIT, debate "Fuzzy versus Conventional Control", 1998 IEEE Conf. on Decision and Control [4]

However, this paper does not intend to make any polemic but it tries to give some thoughts travelling through history. The reader interested in this history can refer to the Lotfi A. Zadeh's paper "Is there a need for fuzzy logic?" published in 2008 [5], that actually embraces a larger area than control.

Let us discuss how the specific area of fuzzy control originated and evolved from such not-too-friendly environment in which, somehow, it is still immersed (see later).

\subsection{The pioneers' heuristic approach: 1965-1985, plus (later spawned) industrial applications}

Initially, fuzzy control was based on natural language and model free synthesis. With three steps (so-called fuzzification, inference, defuzzification), it allowed encoding a nonlinear mapping from the inputs (related to trajectories' reference, measured outputs) to the outputs (control actions) as a controller.

Let us recall therein the pioneering works of Pr Ebrahim Mamdani (passed in 2010) using linguistic synthesis for fuzzy controllers [6]. This heuristic rule-based controller so-called Self-Organizing Controller (SOC) was tested for a long series of experiments on various models, including SISO, MIMO linear, non-linear, with delays, constraints on the inputs/outputs... and had interesting good capabilities [7]. The so-called "expert systems" were nascent in the early 80s and big hopes were put onto them in many disciplines; control problems were one of them [8].

The first industrial application was a cement kiln in Denmark [9] and thousands of them followed in various fields: Washing machines, cameras (Canon), vacuum cleaner (Matsushita)... Micro-waves oven, conditioner (Mitsubishi), showers... Train, lifts, helicopter... A remarkable increase is due to the program LIFE (Laboratory for International Fuzzy Engineering) which took place in Japan in 1988: it included 45 industrials, only one was coming from Europe. 
Let us quote also the first resounding application of the automatic Sendaï metro in Japan [10], which where based on more than 300,000 simulations and 3000 real-time tests improvements for stop precision (divided by 2.5) comfort (increased x2) and consumption (decrease of 10\%) were gained.

Another interesting application was Yamaichi Fuzzy Fund. This is a premier financial application for trading systems. It handled 65 industries and a majority of the stocks listed on Nikkei Dow and consists of approximately 800 fuzzy rules. The system was tested for two years and its performance in terms of the return and growth exceeds the Nikkei average by over $20 \%$ [11]. One of the innovative and successful applications on fuzzy control is helicopter control [12]. After several pioneer and excellent works being presented in the early 1990s, a large number of studies on helicopter control have been conducted as a typical application of UAVs over the last two decades. UAVs are, actually, a popular test bench for modern control and path planning applications.

At last, data compiled for the Berkeley Initiative in Soft Computing (BISC, www.cs.berkeley.edu/ zadeh/ stimfl. html) about patents related to fuzzy logic (therefore larger than control) give the following figures in 2014: number of fuzzy-logic-related patents issued and applied in WIPO (International): 50,999 (USA 22,000; China 25,454; European Union 3,268; Japan 7,100; ...). The reader interested in applications related to the area of fuzzy control can find a recent overview in [13].

\subsection{Decades 1985-2005: the model-based approach}

Pioneering works, despite success stories for applications, suffered from a lack of proofs of key mathematical properties useful for analyzing closed-loop systems: stability, robustness and performances including pole placement, $\mathcal{H}_{2}$ or $\mathcal{H}_{\infty}$ attenuation; such properties were clearly established in the 80 s for linear systems (see, for instance, the celebrated paper [14]).

In this second period, new modelling paradigms for nonlinear systems allowed for such a possibility, with important milestone theoretical achievements. However, the theory-practice gap widened and, basically, the vast majority of today's industrial applications of fuzzy control still rely on the heuristic rulebase ideas conceived in the above earlier period.

\subsubsection{Fuzzy modelling}

In this period model-based approaches took the leading role in developments. Fuzzy models were expressed as a blending of simpler models (linear ones, in the earlier literature) were the representation of choice: consider the linguistic statements

$$
\text { IF } z(t) \text { is } \mathcal{M}_{i} \text { then } \eta=\theta_{f, i} * w_{f}(t)+\theta_{g, i} * w_{g}(t) * u, \quad i=1, \ldots r
$$

where $\mathcal{M}_{i}$ is a (possibly multi-dimensional) fuzzy set (with, maybe, a linguistic label) so that all $\mathcal{M}_{i}$ conform a fuzzy partition (their associated memberships $\mu_{i}(z)$ adding one), $z(t)$ are denoted as premise variables, $\theta_{i}$ are constant model parameters, $\eta$ is the model output (successor state, state derivatives, plant output, etc.), $u$ is the plant input, and $w_{f}(t), w_{g}(t)$ are a vector (or matrix) of known regressors (actually functions of state and inputs, i.e., $\left.w_{f}(t)=w_{f}(z(t), x(t), u(t))\right)$. The linguistic statement was to be understood as

$$
\eta(z, t, u)=\sum_{i=1}^{r} \mu_{i}(z(t))\left(\theta_{f, i} * w_{f}(t)+\theta_{g, i} * w_{g}(t) * u\right)
$$

The values of $\theta_{i}$ for a particular nonlinear plant could be obtained by identification (either via linear leastsquares if $\mathcal{M}_{i}$ were fixed [15], or via non-linear algorithms if $\mathcal{M}_{i}$ had to be also identified [16] by adjusting some tunable parameters of the membership functions).

The so-called Takagi-Sugeno (TS) models [17], in which regressors at the consequent side were functions of the state, have given rise to a plethora of successful fuzzy control designs. TS models are usually written as (only continuous-time discussed, for brevity):

$$
\dot{x}=\sum_{i=1}^{r} \mu_{i}(z(t))\left(A_{i} x+B_{i} u\right), \quad y=\sum_{i=1}^{r} \mu_{i}(z(t))\left(C_{i} x+D_{i} u\right)
$$

and, in most cases, the premise vector is a subset of the state vector, $z \equiv x$. The relationship with linear paremetervarying (LPV) dynamic systems [18] is clear, so these models have also been labelled as quasi-LPV ones [19].

The history of TS fuzzy-model based control goes back more than two decades. The idea began around 1990, when Tanaka and Sugeno published their seminal work [20, 21] introducing fuzzy model construction based on sector nonlinearity (from first-principle models) and parallel distributed compensation (PDC) based on Lyapunov Stability Theory [22]. The basic ideas and some key references will be discussed in Section 1.2.2. 
Regarding the accuracy of the identification-based approach, the universal function approximator paradigm $[23,24]$ allowed to state that (with a high-enough number of rules and regressors in $w_{f}, w_{g}$ ) under very mild assumptions any continuous function could be approximated to any desired degree of accuracy in a compact region. From that early qualitative assesment, the statistical learning theory was perfected and a cohesive treatment emerged dealing with fitting linear, fuzzy, neural and other function approximators from raw data [25].

As a last modelling paradigm, uncertain parameters or nonlinearities can also be cast in the so-called Linear Fractional Transformation form (LFT). This is a well-known modelling task prior to designing robust controllers, see [26]. If the uncertain parameters are known this leads to gain-scheduling LPV solutions; the application of these ideas to the fuzzy modelling context was proposed in [27]. It can be shown that, in some cases, LFT allows representing rational expressions of nonlinearities with a lower number of rules than TS systems.

\subsubsection{Model-based fuzzy control design}

Several tracks have been followed to go from model-free to "classical" model-based designs. First of all, most of the techniques used for nonlinear models can be applied to a TS model (which, obviously, can be seen as a particular case of nonlinear ones): feedback linearization, predictive control, adaptive control, sliding mode control, control based on inverted models... "can" be used with fuzzy models. However, most of these techniques do not explicitly use any specifically "fuzzy" feature of the used models, so using fuzzy models instead of nonlinear ones doesn't provide any particular advantage (and, sometimes, it has disadvantages). In a reverse case, interpolation steps needed in other model-based techniques were proposed with fuzzy rules, for instance in sliding fuzzy control in which a fuzzy rulebase can be used to interpolate the (hard) switching surface arising in sliding-mode control to avoid chattering; however such fuzzification provides nothing significantly different to other options such as plain linear interpolation [28].

A particular case of the above use of fuzzy models for other nonlinear tools is the following: as a nonlinear map $\dot{x}=f(x)$ can be expressed as the feedback interconnection between the linear system $\dot{x}=A x+\eta$ and the non-linear static one $\eta=f(x)-A x$, stability of TS systems (or that of linear systems with an static heuristic fuzzy controller) can be evaluated via reformulations of classical linear-nonlinear feedback results such as circle, Popov or frequency response (descriptive function) ideas, or multivariable generalisation of them (conicity). This approach is pursued in the early works $[29,30]$ and some indices were also derived, based on Jacobian matrix, to indicate how far from instability a fuzzy loop is [31, 32].

These results in the 90 s were valuable, in the sense that they were a first step to prove stability of the "heuristic" controllers of a nonlinear plant in a neighbourhood of the equilibrium, or that of some TS systems, but the approach (linear plant plus static nonlinearity) didn't provide flexible enough criteria for design and did not consider performance measures such as $\mathcal{H}_{\infty}$ bounds under disturbances. Indeed, by that time, optimal $\mathcal{H}_{2}$ and $\mathcal{H}_{\infty}$ designs for linear systems were well established, so there was a need to generalise such results to a non-linear setting in a clearer way.

In that decade, the seminal linear matrix inequality (LMI) approach [33] for linear and uncertain systems was quickly adopted in the fuzzy control community. In particular, there has been a flurry of research activities on LMI-based fuzzy approaches after the remarkable work [34, 35] was presented; the well-known book [22] summarises many key contributions in that respect in the 90s. The LMI approach to control was a "revolution" in the 90's in many aspects of control. The Riccati Equations for linear systems the 80's were replaced by LMI constraints in many extensions of the classical concepts to linear-nonlinear-uncertain-fuzzy-local-... stability and control design problems. Given the existence of efficient convex-optimisation based software [36] to solve them, since mid 1990's, a control problem was considered solved once expressed as a set of LMIs; anyway, it is worth noting that most of such LMIs actually pose only sufficient conditions for solving the problem (if feasible).

Indeed, the basic idea was posing controllers for (2) in the form $u=\sum \mu_{i}(z) F_{i} x$ (denoted as parallel distributed compensators, PDC), yielding closed loops in the form $\dot{x}=\sum_{i=1}^{r} \sum_{j=1}^{r} \mu_{i}(z) \mu_{j}(z)\left(A_{i}+B_{i} F_{j}\right)$, where the right-hand-side term in the expression of $\dot{x}$ conforms a so-called 2-dimensional fuzzy summation. From these expressions, most fuzzy control results ended up in the need of proving a matrix inequality:

$$
\sum_{i=1}^{r} \sum_{j=1}^{r} \mu_{i} \mu_{j} \Xi_{i j}>0
$$

for which some shape-independent ${ }^{1}$ relaxations in LMI form could be written. The simplest of it is, trivially, $\Xi_{i j}+\Xi_{j i}>0$. For instance, based on that relaxation, an straightforward LMI to prove quadratic stability for state

\footnotetext{
${ }^{1}$ Shape-independence entails proving (3) for any positive $\mu_{i}$ as long as they add one, instead of the specific "shape" $\mu_{i}(z)$; of course, this might involve a lot of conservatism.
} 
feedback is $\left(A_{i} P+B_{i} M_{j}+A_{j} P+B_{j} M_{i}\right)+\left(A_{i} P+B_{i} M_{j}+A_{j} P+B_{j} M_{i}\right)^{\prime}>0$ for all $1 \leq i \leq j \leq r$. If feasible, the PDC controller gains are $F_{i}=M_{i} P^{-1}$.

Of course, apart from the above straightforward controller, other more sophisticated Lyapunov functions and control laws were also proposed in this exciting period. By 2005, there existed a large body of literature on stability analysis and design of T-S fuzzy control systems. Improvements have appeared in piecewise/gridpoint approaches [37, 38], non-PDC design [39, 40, 41], multi-step non-monotonic Lyapunov functions [42], and LinearFractional transformation approaches to fuzzy modelling [27], with clear links to the LPV gain-scheduling concepts [18]. Widely used relaxations of the double-summation problem (which apply to many fuzzy results) appeared in $[43,44]$, although conservatism remained.

Also, straightforwardly, the T-S models were extended with time-delays. As in the linear/robust case, stability of T-S delay systems can be naturally classified into two classes: delay-independent and delay-dependent. Naturally the second class is less conservative than the first one, considering that information on the delays is taken into account: the class of systems that can withstand an arbitrarily long delay is very reduced.

Pioneering works used Lyapunov-Razumikhin functional approaches for stability and stabilization problems [45] and mainly limited stabilization to the delay- independent case. Delay-dependent conditions appeared as a continuation, introducing classical Lyapunov-Krasovskii functional (LKF) methods for example in [46, 47, 48] or using descriptor representation [49]. These works initiated a lot of tracks to be further developed.

So, after the seminal theoretical developments in the period, the situation in the area of fuzzy systems and control got characterized by a striking mismatch between, on one hand, one of the main motivation of fuzziness, that is, readability (using understandable rules, computing with words) and, on the other hand, the use of mathematically involved and non-transparent techniques (optimisation on hundreds of variables) to ensure robust performance, in direct analogy with mainstream (nonlinear and gain-scheduled) control theory. From a research point of view, in the low-level control loop (models with differential equations) the knowledge-based approach was basically superseded by the model-based one in that time period. From the late 90's onwards, the original "intelligent control" knowledge-based approach only remains an option in higher control levels (supervision, diagnosis, planning,...) dealing with "qualitative" concepts instead of differential equations.

\subsection{Adaptive fuzzy control}

Adaptive fuzzy control deals with using input-output data in order to craft suitable update laws to change process or controller parameters $\theta$ in time, using process models and controllers in the form (1). Linear adaptive control was actually in its infancy in most of the earlier period (pre-1985) and, hence, no significant contributions in incorporating fuzzy ideas to adaptive control were produced until the 1990's.

The SISO/MIMO nonlinear systems were usually considered in the canonical Brunowski form $y^{(r)}=f(x)+$ $g(x) u$, being $y^{(r)}$ an output's $r$-th time derivative, with $f(x)$ and $g(x)$ (the latter one assumed regular, a controllabilitylike assumption) being unknown nonlinear functions of appropriate size and $r$ being the relative degree.

Based on the universal function approximator, such functions are expressed as (1) (plus an error term tending to zero as the number of regressors grows) [50]. Two different approaches apply: direct and indirect adaptive control. In the direct method the fuzzy system directly approximates the "ideal" control law, and its parameters are tuned directly to meet the objectives [51]. The indirect scheme was based on classical feedback linearisation, i.e., using the ideal unknown control law $u=g^{-1}(x)(v-f(x))$ with $v$ a new control vector so $y^{(r)}=v$, and setting $v=y_{m}^{r}+K e$ being $y_{m}$ the tracking reference and $K$ a stabilising linear state feedback gain for a chain of $r$ integrators. Instead of the unknown $f$ and $g$, generally, two parameterized approximation $\hat{f}\left(x, \theta_{f}\right)$ and $\hat{g}\left(x, \theta_{g}\right)$ of $f(x)$ and $g(x)$ respectively, are used, being $\theta_{f}$ and $\theta_{g}$ the estimated parameters; widely-cited references of that period are $[50,52,53]$. In fact, given that many results rely on the universal approximation property and the basic difference is in the approximator being linear or not in the adjustable parameters, many fuzzy, neural and classical adaptive approaches of the decade may be viewed under a unifying perspective [54].

These different methods suffered from several limitations. Amongst them, real time feasibility, computational cost, bounding parameters values [55] and the major one was the difficulty to go from state-feedback adaptive control (state is assumed measured) to output-feedback control, addressed in [56]. This last point had several solutions but majority of them suffered from drawbacks, a useful review and critics of these first proposals is given in [57].

\section{What did the last decade bring us: (2005-present)}

Amongst these various approaches in the last 10 years it appears that the principal results focused mainly on two techniques: model based control using the so-called Takagi-Sugeno quasi-LPV models and adaptive control using the property of universal approximation of the fuzzy models. Also, a new paradigm based on polynomial fuzzy models appeared. Therefore, the overview will mainly focus on these three approaches, pinpointing a few 
key developments based on the authors' interest and experience but, maybe, leaving out significant results in the contribution of fuzzy modelling and control to stochastic, Markov, PDE systems, higher-level planning, robotics, etc.

\subsection{New improvements in TS system analysis}

Regarding TS systems analysis, one of the leitmotivs of the last decade was to develop the non-quadratic Lyapunov function ideas first introduced at the end of the previously revised period. Another source of results is extending prior results to nonlinear systems with delay, stochastic inputs or parameter variations, etc. Many of the stability/stabilization results can be translated to various performance possibilities (decay, $\mathcal{H}_{2}$ guaranteed cost, $\mathcal{H}_{\infty}$, robust stabilization, ... ). Let us briefly discuss some of the main ideas.

First, a brief mention to a basic result: in order to "decouple" decision variables in control gains from those in Lyapunov functions, many of the results make heavy use of the so-called Finsler's lemma. Consider a vector $\psi$ and a Lyapunov inequality $\psi^{T} T_{z} \psi<0$ which must be fulfilled only when some model equality holds $H_{z} \psi=0$, where subindex $z$ denotes a dependence on the membership functions. Then, if a multiplier $M_{z}$ can be found such that $T_{z}+M_{z} H_{z}+H_{z}^{T} M_{z}^{T} \leq 0$, the constrained inequality is proven to hold. Lyapunov decision variables appear in $T_{z}$, and controller gains appear in $H_{z}$. A suitable choice of multiplier (albeit, in most cases, conservative) can write the problem as LMI or, in some cases, as an LMI plus some scalars to be searched for.

\subsubsection{Non-quadratic Lyapunov functions}

Consider a generic Lyapunov function (usually $V(x)=x^{T} P(\cdot) x$ for observation or $V(x)=x^{T} P^{-1}(\cdot) x$ for control with the actual arguments and structure of $P(\cdot)$ to be defined according to the case under study. A constant $P(\cdot)$ corresponds to the quadratic case.

Mainly, the first parametrizations of $P(\cdot)$ were variations of the form $P(\cdot)=P_{z}=\sum_{i=1}^{r} \mu_{i}(z(t)) P_{i}$, the so called "multiple" or "fuzzy" Lyapunov function [40, 41], commented in the previous section. Multiple-sum Lyapunov functions, in the form $P(z)=\sum_{i_{1}=1}^{r} \sum_{i_{2}=1}^{r} \cdots \sum_{i_{d}=1}^{r} \mu_{i_{1}}(z(t)) \ldots \mu_{i_{d}}(z(t)) P_{i_{1} i_{2} \ldots i_{d}}$ were proposed afterwards in $[58,59,60]$. Accordingly, multiple sums were introduced in the controller and observer gains. Results were less conservative as the number of sums involved increased, see Section 2.4. Another interesting result was the so-called $\alpha$-samples variation, based on the following idea: in an asymptotically stable system the states tends to zero as time increases, so if initial conditions lie at any level set of an arbitrary strictly positive-definite function $\tilde{V}(x), \tilde{V}(0)=0$, the state will eventually enter any inner level set, even if $\tilde{V}(x)$ is not a "true" Lyapunov function itself (i.e., even if the one-step variation $\tilde{V}(x(t+1))-\tilde{V}(x(t))<0$ cannot be proved). This allows replacing the standard one-step variation by an $\alpha$-sample variation $\tilde{V}(x(t+\alpha))-\tilde{V}(x(t))<0$ and still have a sufficient condition to prove stability [61], see details in [62]; conservatism reduces as $\alpha$ increases. The idea was extended to general Lyapunov functions and control/observer structures in [63, 64, 65].

A renewed interest in the continuous-time non-quadratic stabilization also appeared, in three directions.

A first family of results deals with the assumption, originally stated in [40], of bounded membership-function time derivatives, i.e., existence of known scalars $\phi_{i}$ such that $\left|\dot{\mu}_{i}(z)\right| \leq \phi_{i}$. Results in this line appear in, for instance, [66]. Although the assumption is understandable for stability results, when $z \equiv x$, which is the case in nonlinear systems modelled as TS ones, it is more questionable for stabilization as, by chain rule, $\frac{d \mu_{i}(x)}{d t}={\frac{\partial \mu_{i}}{\partial x}}^{T} \dot{x}$ and $\dot{x}$ can contain the to-be-designed control action in a general case, so the validity region of the obtained controller must be checked a posteriori, see discussion in [67]. In [68], the parameter-dependent (a.k.a. fuzzy) Lyapunov function is exploited for stabilization with output-feedback gain-scheduled controller for linear-fractionaltransformation descriptions; the resulting controller needs real-time measurements of the parameters (memberships) and their derivative.

As an alternative to bounding $\dot{\mu}_{i}$, a nice global result without such bounds appeared in [69], exploiting the so-called path-independent Lyapunov property, but with two key restrictions: a limitation to a specific class of TS models and BMI formulation in the stabilization case. A refinement based on slack variables can be found in [70], that gives, under some assumptions, parameter-dependent LMI problems.

The third option leads to a family of results proposing recasting the problem from global stability with timederivative bounds (first idea above) to local stability with bounds on the partial derivative of the memberships. The basic idea is that, if a bound $\left\|\frac{\partial \mu_{i}}{\partial x}\right\| \leq \gamma_{i}$ exists, with known $\gamma_{i}$, then $\left|\dot{\mu}_{i}\right| \leq \gamma_{i}\|\dot{x}\|$ so LMIs to ensure control gains are chosen bounding $\|\dot{x}\| \leq \beta_{i}$ in a particular Lyapunov level set can be crafted; subsequently, Lyapunov decrease conditions where $\beta_{i}$ and $\gamma_{i}$ appear can be stated. If feasible, local stabilisation is guaranteed in the modelled region without the need of a posteriori checks. The idea has been developed in [71, 67] and embedded into multiple-sum Lyapunov functions in $[72,73]$. 


\subsubsection{Delay}

Initiated in the first years of the past decade, works for TS time-delays systems have increased in an important proportion in the last 10 years. Delay analysis involves use of Lyapunov-Krasovskii functionals in the form $V=$ $x^{T} P x+\int_{a}^{b} x^{T} Q x+\int_{c}^{d} \dot{x} R \dot{x}+\int_{e}^{f} \int_{g}^{h} \dot{x} S \dot{x}+\ldots$ for some delay-bound related integration limits. Also, Finsler lemma is routinely used to replace integral terms in $\dot{V}$ by delayed states, as $M_{z}\left[x(t)-x(t-\tau)-\int_{t-\tau}^{t} \dot{x}(s) d s\right]=0$, for any arbitrary multiplier expression $M_{z}$, jointly with Jensen's inequality and other technical lemmas.

In 2006, [74] proposed the introduction of extended Lyapunov-Krasovskii functional (LKF) that copes with interval time-varying delays removing any assumption on the derivative of time-delays. Then, the efforts to reduce the conservativeness of the results mainly resumes in constructing new augmented LKF approaches. For example in [75] a triple integral form associated with the so-called Finsler's lemma and some augmented vectors including further information of time-varying delays are exploited. Nevertheless this onward rush led quickly to computational problems due to a huge increase in the number of decision variables. Another idea developed concerns delay-partitioning approach for interval time-varying delays with the idea not only to outperform the methods but also to reduce the number of LMI constraints as well as the number of decision variables in comparison with previous approaches $[76,77]$. At last, a new way of writing the positiveness of the LKF, i.e. not every term of its sum is needed to be positive can be found in [78]. Most of these interesting results mainly focus on stability problems with state delays. Therefore, there is room for a lot of combinations to work on: state feedback [74] using the input/output framework via interconnection schemes and scaled small-gain condition [79], state feedback with inputs delays [80], output feedback $\mathcal{H}_{\infty}$ control design [81] and many others (out of the scope of this overview).

Some attention seems to be paid, too, to stochastic fuzzy systems where Ito's differential formula [82] is used instead of the standard time derivative to account for variance effects when taking the derivative of the average of a quadratic form. Combination of such systems with delay ones appear in, for instance, [83, 84, 85].

\subsection{Fuzzy-polynomial techniques}

From the discussion on section 1.2, the success history of Takagi-Sugeno (T-S) fuzzy-model based control goes back more than two decades.

Though LMI-based approaches have enjoyed great success and popularity, there still exist a large number of design problems that either cannot be represented in terms of LMIs, or the results obtained through LMIs are sometimes conservative. A post-LMI framework is the Sum-of-Squares (SOS) approach. The basic idea of the SOS approach is realising that any degree $d$ polynomial $p(x)$ can be written, if $d$ is even, in the form $\psi^{T}(x) R \psi(x)$ with $\psi(x)$ being a vector of all the monomials up to degree $d / 2$ and $R$ a (non unique) matrix trivially built from the polynomial coefficients. Maximising the minimum eigenvalue of $R$ over the set of matrices such that $\psi^{T}(x) R \psi(x)=p(x)$ is an LMI problem, as $R$ ranges in an affine subspace. If a positive minimum eigenvalue is found, then $R>0$ and the Choleski factor of it, say $Q$, allows expressing $p(x)=(Q \psi)^{T}(Q \psi)$, i.e., a sum of squares. So finding SOS decompositions of a polynomial is a convex problem and LMI Lyapunov results (degree 2 polynomials) can be easily extended to higher degree polynomials in both Lyapunov function and process model [86]. Another key result (Positivstellensatz) extending the S-procedure is the fact that $p(x)>0$ for all $x$ such that $q(x)>0$ if there exists a SOS multiplier $\mu(x)$ such that $p(x)-\mu(x) q(x)$ is SOS, allowing to prove local positiveness of polynomials. Note, however that there are positive polynomials which cannot be written as a sum of squares [87]. When the multiplier doesn't need to be SOS, the concept extends Finsler's lemma (Section 2.1) to polynomial equality constraints.

The extension to fuzzy polynomial models in the form $\dot{x}=\sum_{i=1}^{r} h_{i}(z(t))\left(p_{i}(x)+q_{i}(x) u\right)$, with $p_{i}, q_{i}$ polynomial matrices, was first proposed in 2007 [88, 89, 90]. Such polynomial fuzzy models include the well-known Takagi-Sugeno fuzzy systems and controllers as special cases. In fact, the above models, by extracting common polynomial factors, are usually expressed in a more TS-looking way as: $\dot{x}=\sum_{i=1}^{r} h_{i}\left(A_{i}(x) \zeta(x)+B_{i}(x) u\right)$, where $\zeta(x)$ is a vector of monomials.

An efficient polynomial fuzzy model construction, from nonlinear first-principle ones, based on Taylor series approach has been proposed by extending the sector nonlinearity idea in [91]; it can reduce conservatism (in system design and analysis) caused by fuzzy model construction with respect to TS approaches. The SOS approach has given rise to later developments in control [92, 93], observers [94], fuzzy polynomial Lyapunov functions [95], both in continuous-time and in discrete-time [96]. Using the above mentioned multipliers, conservatism in local stability results can be reduced $[93,97]$. Polynomial and delay or sample-data techniques can be, too, combined [98]. In fact, many TS results with an LMI involving constant vertices $A_{i}$ and Lyapunov matrix $P$ can be translated to polynomial-fuzzy with $A_{i}(x)$ and $P(\hat{x})$ via straightforward developments (note that $\hat{x}$ cannot be the full state $x$ in some cases, see below): the standard $V(x)=x^{T} P x$ is translated to $V=\zeta(x)^{T} P(\hat{x}) \zeta(x)$. Denoting as $J_{\zeta}$ the Jacobian matrix of $\zeta$, we have

$$
\dot{V}=2 \zeta(x)^{T} P(x) J_{\zeta} \dot{x}+\zeta(x)^{T} \dot{P}(\hat{x}) \zeta(x)=\sum_{i} h_{i} \zeta(x)^{T}\left(P J_{\zeta} A_{i}+A_{i}^{T} J_{\zeta}^{T} P\right) \zeta+\zeta(x)^{T} \dot{P}(\hat{x}) \zeta(x)
$$


where a "standard" translation $\left(P J_{\zeta} A_{i}+A_{i}^{T} J_{\zeta}^{T} P\right)$ of the usual Lyapunov derivative appears, but adding $J_{\zeta}$ in the middle as a consequence of chain-rule. Regarding the rightmost term, choosing the argument of $P(\hat{x})$ in stability analysis as $\hat{x} \equiv x$, then $\dot{P}(x)=\sum_{l=1}^{m} \frac{d P}{d x_{l}} \dot{x}_{l}$ is a polynomial-fuzzy matrix. In control synthesis $\hat{x}=E x_{k}$ is chosen, with $E$ such that $E B_{i}(x)=0$ for all $i$ (in this way, no control-related decision variables appear in $\dot{P}(\hat{x}) ; V(x)$ in inverse form $\zeta^{T} P^{-1}(\hat{x}) \zeta$ is also helpful) and, as intuitively expected, $A_{i}(x)$ should be replaced by $A_{i}(x)+B_{i}(x) F_{j}(x)$. Details are omitted for brevity, but many TS results (stabilisation, guaranteed-cost, $\mathcal{H}_{\infty}$, delay,... ) can be "polynomialised" developing the above idea (plus suitable Positivstellensatz multipliers for local stability, if so wished).

\subsection{Shape-dependent laws}

Expressions (3) are, in general, proved via LMI sufficient conditions in the sense that positiveness is proved for any value of $\mu$ in the standard simplex (memberships positive and adding one). Of course, if successful then the conditions for a particular "shape" $\mu_{i}(x)$ arising from modelling a particular system to be controlled do hold, but they also hold for a large family of related plants which actually should not be controlled. This is the so-called shape-independent conservatism: shape-independence plugs robustness in places we might not actually want to.

Once the issue was realised, some contributions in that respect were developed: Relaxations including knowledge that a particular set of linear expressions of the memberships held true [99], generalisation of such a case to polynomial constraints on the memberships [100, 101], NQ Lyapunov functions from bounds in partial derivatives of memberships [67], combined membership-state polynomial constraints [102], etc. Also, in most modelling problems in which nonlinearities are separately modelled by interpolation between two bounds, the resulting membership functions have tensor-product structure; exploiting this allows reduced conservatism [103, 104]. Shapedependent analysis with TS fuzzy models of the partial derivatives of membership functions appears in [95].

\subsection{Asymptotical exactness}

Several results in the last decade, some of them already mentioned above, have achieved the so-called asymptotical exactness, in the following sense: conservatism reduces as used computational resources increase (modulated by a so-called complexity parameter) and, in theory, when the complexity parameter of the proposed algorithms tends to infinity, conservatism reduces to zero (well, usually only in a particular facet of it). Such results are of theoretical interest, in the sense that they "close" important problems which were open 10 years ago. However, regarding the practical side, usually the increase of computational resources is exponential (or combinatorial) in the complexity parameter, so a very limited improvement over clever existing solutions can be achieved, except in simple cases. Let us briefly discuss the main ideas in some of these "asymptotically exact" results:

Fuzzy summations. A set of convex problems proving shape-independent positive-definiteness of expression (3) with reduced conservativeness was first proposed in [105] and a related triangulation-based approach appeared in [106]. In fact, SOS versions of such relaxation can also be conceived [107]. The basic idea is the so called matrixPolya theorem: if $\Xi=\sum_{i_{1}=1}^{r} \sum_{i_{2}=1}^{r} h_{i_{1}} h_{i_{2}} Q_{i_{1} i_{2}} \geq \gamma>0$ then there exists a complexity parameter $d$ such that, from the equivalent expression $\Xi=\left(\sum_{l=1}^{r} h_{l}\right)^{d} \sum_{i_{1}=1}^{r} \sum_{i_{2}=1}^{r} h_{i_{1}} h_{i_{2}} Q_{i_{1} i_{2}}>0$, expressing $\Xi$ as a matrix polynomial in $h$ (grouping all elements which are multiplied by the same monomial in $h$, up to degree $d+2$ ) all its coefficients are positive definite matrices; the reader is referred to the cited references for details. The term $\Xi$ is denoted as a multi-dimensional fuzzy summation.

Lyapunov function families. Reaching any possible smooth Lyapunov function using the above multi-dimensional fuzzy summations in fuzzy Lyapunov functions [59], or increasing the degree of a polynomial Lyapunov function at will [108, 101] progressively increases the expressive power of the chosen Lyapunov function family. In the polynomial case, if there exists a smooth Lyapunov function (so that its Taylor series converges to it), the result is asymptotically exact as degree increases (well, actually, up to the gap between positive and SOS polynomials [87]).

There are other ideas which allow generalising the concept of Lyapunov functions, or even "dismissing" it (proving stability without having explicitly found such function). A first proposal is the $\alpha$-samples argumentation outlined in Section 2.1.1: from the sheer definition of stability, if you wait long enough, any non-degenerate quadratic function is an $\alpha$-sample Lyapunov function for any asymptotically stable system, even if it is not a "standard" Lyapunov function; based on that, the work [62] proposed discrete-time generalisations asymptotically exact as a delay parameter increases. A second approach uses necessary and sufficient shape-independent stability conditions for polytopic linear differential inclusions (i.e., TS systems in the fuzzy jargon) systems. These conditions can be stated with a Lyapunov function chosen from a family of either polyhedral, piecewise quadratic or convex-hull of ellipsoids [109]: a constructive algorithm to prove the largest possible shape-independent domain 
of attraction (maximal invariant set in non-fuzzy literature) of a TS system is proposed in [110]; such set is computed by an iterative algorithm (which converges in a finite number of steps, under mild assumptions), producing a polyhedral Lyapunov function whose existence is asymptotically necessary and sufficient for stability of the TS system (up to a Polya-fuzzy-summation complexity parameter); asymptotic exactness can also be proven for the stabilization problem [111], although the computational burden is higher.

\subsection{Adaptive control}

Since the starting steps discussed in Section 1.3, several issues have been addressed in the past decade. One issue is that the number of parameters to be tuned in the online control laws in such methods is too important; therefore both the learning time and the real time feasibility are rapidly faced to problems when dealing with high order systems. Some proposals have been done; amongst others using lower triangular structure, thus mixing with backstepping techniques, for indirect scheme [112], for direct scheme [113].

Another issue that has been tackled is when there is no a priori knowledge about the sign of the control gain. Solutions were obtained using the so-called Nussbaum-gain technique [114]. To avoid the measurement of the whole state, and go to output feedback control, several (direct and indirect) adaptive fuzzy observer design schemes have also been proposed [57, 115]. Especially in [57], for SISO systems the design is done for any kind of observer structure (linear, high gain, sliding mode) and both the update law and the robust term are based on filtering the output tracking error. The interest is therefore to overcome the strictly positive real condition.

At last some refinements mainly dealing with approximation-based adaptive backstepping control were also derived, for example MIMO systems with unknown coefficient gain signs [115], MIMO time-delay systems with nonlinearities on the inputs [116], with dead-zone [117] and recently MIMO systems with input saturations and output constraints [118].

\section{General discussion about present and future}

As a general thought, the last decade has brought a better understanding of the conservatism inherent to fuzzy approaches to nonlinear control. So, now, we have a family of powerful conditions on Lyapunov function choices, polynomial modelling, non-quadratic Lyapunov functions, summation relaxation which, however, hit current computer's limits with low-order state-feedback problems. Problems with high-order systems, or output feedback (even harder with non-measurable premises), haven't improved so much in practical terms in the last decade.

Given the asymptotical exactness of some solutions (with, however, unsurmountable computational resources), the conceptual picture of the situation appears below:

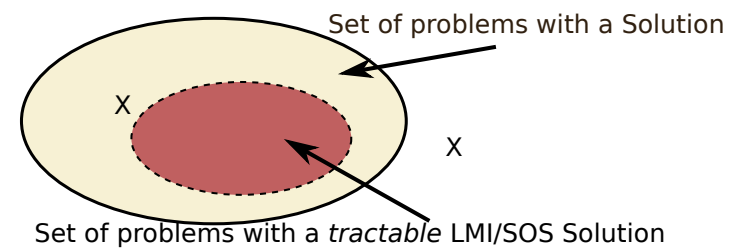

If the white ellipse represents the unknown set of problem with a solution, whose determination is computationally very hard, the general goal is twofold. First, necessary conditions to exclude unfeasible problems (cross mark out of the set) are needed (for instance, to determine that there does not exist a controller satisfying a particular performance goal), second computationally efficient solutions must be found to outperform current sufficient conditions to enlarge the red area and capture more feasible points (cross mark into the set).

For the first point very few results are available [106]. Most of the results try to solve the second problem (indeed, solutions outside the red set can be proved neither feasible nor unfeasible but, taking the worst case, they are usually dismissed).

\subsection{Old problems still unsolved}

Some problems which were present since the origins of the sector-nonlinearity LMI approach have not been overcome (and possibly, they will never $\mathrm{be}^{2}$, because of the sheer philosophical idea of fuzzy models: instead of $\dot{x}=f(x)$ we change in TS modelling to $\dot{x} \in F(x)$ where $F(x)$ is a set with either a finite number of vertices (equal to the number of rules, in open-loop) or with an infinitude of them (in closed-loop, due to multiple-summations;

\footnotetext{
${ }^{2}$ At least in a membership-shape-independent approach. Of course, as TS models are equivalent to the original nonlinear ones, ideally an infinitely-powerful shape-dependent approach could operate on the "true" nonlinear system without conservatism. Such approach, however, is currently non-existent within the TS modelling framework.
} 
sum-relaxation embed such set in a polyhedron with an ever increasing precision). Then, many key geometrical aspects of the nonlinear system is lost under the "cloud" of points which comprise the image of a single $x$, because differential geometry cannot be carried out in set-valued maps. Observability, controllability, feedback linearisation results (which are, sometimes, straightforward in an original nonlinear model) get cumbersome (or, plainly, impossible) in the TS representation of it. In the same way, decoupling, inversion and two-degree-of-freedom control, frequency-response (descriptive function), interaction measures are also hard to deal with in the TS platform, being, however, of paramount importance in (linear) multivariable process control applications in industry [26].

Other key issues, for which no truly significant achievement has been proposed in the last 10 years, lie in the modelling and identification arenas. Regarding fuzzy modelling of complex systems by sector-nonlinearity approaches, as each nonlinearity gives rise to two rules, the number of rules is a power of two and some of the results' computational requirements increase hugely with the number of nonlinearities (even in the TS approach), and the model is non-unique. Again, although theoretically elegant, no more than 4 or 8 rules can be reliably assessed in the latest high-order fuzzy/polynomial/local results. Fuzzy identification, set up as solving optimisation problems involving minimising the squared prediction error, hasn't advanced that much recently; as in 2005, there is no "cross-talk" yet between the control requirements (usually in terms of model error at certain target "bandwidth", uncertainty written in particular LFT form) to the key issues of learning/identification of dynamic systems (experiment design, number of data needed, speed of convergence of algorithms, local minima, etc.).

\subsection{An appraisal of the present situation}

We can reuse the question of [5] "Is there a need for fuzzy logic?" and go for "Is there a need for fuzzy control?". Indeed, there are many debatable points out there deserving some discussion.

Industry reach. Linguistic approaches are clearly hopeless in future research, albeit industry associations promoted fuzzy rulebases to be integrated into industrial automation standards (IEC 61131-7) more than 10 years ago. However, even if extremely useful, current linguistic fuzzy applications in industry seem to be only ad-hoc refinements to linear regulators or substitution for coarse-grained lookup tables, or soft thresholds for alarms. Nonlinear predictive control seems to have won the match regarding transition from theory to industry [119] in advanced nonlinear control problems, due to the inherent optimisation embedded into it, and the above-mentioned lack of an elegant way of handling inversion/decoupling/bandwidth issues in the TS approach.

“Fuzzy” versus "linear parameter-varying” versus "nonlinear". As shown throughout this paper, current fuzzy control approaches are more or less entering in the "classical" automatic control way of doing: the "fuzzy" term loses some of its historic interest, such as taking into account "vagueness" and "uncertainty". The robust control way of defining and dealing with uncertainty has been the way to go since LMIs were around. Nowadays, the communities of linear parameter varying (LPV), quasi-LPV and TS (basically, different names for the same thing), as well as the publications are getting close. Indeed, at the end, fuzzy is a means to actual control of nonlinear systems by linear-like techniques. Linear time-varying approaches are also a means to do that and shape-independent fuzzy approaches are somehow philosophically similar to LTV analysis, as the relationship between premise variables and state variables is not actually exploited.

Ideally, only shape-dependence involving combined state/membership constraints would actually be truly "nonlinear" (non-conservative); otherwise, if $\mu(z)$ are handled as variables independent from $x$ in stability conditions, there is no "key" difference with a linear time varying $\sum_{i} \mu_{i}(t)\left(A_{i} x+B_{i} u\right)$ model. Bounding the timederivative of memberships is not "so nonlinear", in the sense that $\dot{\mu}_{i}(t)$ has also been considered by the LTV people since at least the mid-90's. Recent contributions bounding the partial derivative of the memberships with respect to state (not time) feels philosophically more on the right track as the decisive fact that memberships are functions of the state is implicitly assumed. The advent of SOS (allowing analysis of fuzzy-polynomial systems) has further moved fuzzy control towards the mainstream nonlinear landscape.

Scientific impact of fuzzy control. It is difficult to evaluate the real impact of fuzzy in control through all literature. Nevertheless it really exists; as an example, two main international journals are directly concerned with it: Fuzzy Sets and Systems and IEEE Trans. on Fuzzy Systems. A Technical Committee "Computational Intelligence in Control" from the International Federation of Automatic Control (IFAC) is also devoted to these approaches.

However, the initial aversion towards the heuristic fuzzy approach in earlier time still distills disliking towards fuzziness in prominent control journals. Indeed, substandard/heuristic fuzzy control papers appear (elsewhere) with too high a frequency and get indexed in major databases. The visibility of such contributions doesn't give good reputation.

So, how does fuzzy control keep up in "top" journals? Well, it depends... Very roughly speaking, searching for the keyword "fuzzy" in selected top "control theory" journals brings up around 40 times less results than searching 
for "control" in top "fuzzy" journals. However, alike concepts appear in the former ones under polytopic or gainscheduling denominations, and some "classical" people sends some results to fuzzy journals, too. Anyway, there is still a clear asymmetry and more cross-talk should be promoted.

Other approaches. Some different tracks are tested nowadays that are (in our opinion) more or less successful: type-II fuzzy sets, neuro-fuzzy, interval approaches extend to fuzzy sets, ...

For instance, some control results with the so-called Type-2 sets (where membership function $h_{i}(x)$ is itself a fuzzy set) claim superiority over type-1 or conventional linear controllers (for instance,[120]); however, given that conventional (type-1) fuzzy models are universal function approximators, there is no type-2 controller which cannot be crafted with standard type-1 setups: so, the superiority claims do not have a firm theoretical support for the time being. A more focused approach to type-2 control, as a way to analyse uncertain nonlinear systems, might be interval approaches pursued in, for instance, [121]. However, the underlying idea is, philosophically, related to older literature in which "imperfect knowledge of the plant memberships" is assumed, such as [122].

\subsection{What should, could be the future?}

From the above discussion, at last, several questions arise: how can we come back to the "fundamentals" of fuzzy logic? Is there still space for a "linguistic" way of doing? Or are we, in a sense, loosing the "essence" of what fuzziness was created to?

Of course, fuzzy control should also go to the areas where mainstream automatic control is moving to, among them, large scale systems, interconnected (networks) large systems, hybrid systems... Evolutionary results (juxtapostion of prior large-scale, networked, polynomial-delay, polynomial-networked, ... results) are indeed expected; however, some of them will be so computationally demanding than they will be useful only for "large-scale" systems comprised of "a couple of 2nd-order systems". Also, proving that something with a very involved theory works in noiseless state-feedback isn't that useful in practice, by the way.

Only "straightforward in theory" plus "straightforward in practice" are "great" solutions to change a discipline... Somehow, the earlier fuzzy controllers (with its limitations) were a bit like that (considered in their historical context, i.e., in the 70's); the LMI approach spurred a revolution in the 90's when problems previously thought as intractable were solved in fractions of a second with ten lines of code... On the contrary, even if theoretically elegant, practical computational aspects of the fuzzy-polynomial approach make it not as promising as it was thought to be 8 years ago...

Well, maybe, we are waiting for the next "big" thing in fuzzy control... such revolutionary things are, of course, always unexpected. And, of course, there will always be the beauty of some theoretical concepts irrespective of whether they are of any practical use. It seems that, for theoretical aspects, we are at a crossroad where some new interesting "step" has to emerge to go further than just some adjustments in various known techniques in order to confidently say "yes, there is a need for fuzzy control" in the future.

Only those who attempt the absurd... will achieve the impossible. I think... I think it's in my basement... Let me go upstairs and check. (Maurits Cornelis Escher, 1898-1972)

The truth is rarely pure and never simple.

(Oscar Wilde, 1854-1900)

\section{Bibliography}

[1] A. Sala, T. Guerra, R. Babuška, Perspectives of fuzzy systems and control, Fuzzy Sets and Systems 156 (3) (2005) 432-444.

[2] L. Zadeh, My life and work - a retrospective, Appl. Comput. Math. (2011) 4-9.

[3] B. Kosko, Fuzzy thinking: the new science of fuzzy logic, Hyperion New York, 1993.

[4] D. Y. Abramovitch, L. G. Bushnell, Report on the fuzzy versus conventional control debate, IEEE Control Systems Magazine 19 (3) (1999) 88-91.

[5] L. A. Zadeh, Is there a need for fuzzy logic?, Information Sciences 178 (13) (2008) 2751-2779.

[6] E. H. Mamdani, S. Assilian, An experiment in linguistic synthesis with a fuzzy logic controller, International journal of man-machine studies 7 (1) (1975) 1-13.

[7] T. J. Procyk, E. H. Mamdani, A linguistic self-organizing process controller, Automatica 15 (1) (1979) $15-30$. 
[8] K. J. Åström, J. J. Anton, K.-E. Årzén, Expert control, Automatica 22 (3) (1986) 277-286.

[9] L. Holmblad, J. Ostergaard, Control of a cement kiln by fuzzy logic, in: M. Gupta, E. Sanchez (Eds.), Fuzzy Information and Decision Processes, North-Holland, Amsterdam, 1982, pp. 389-399.

[10] S. Yasunobu, S. Miyamoto, Automatic train operation system by predictive fuzzy control, Industrial applications of fuzzy control 1 (18) (1985) 1-18.

[11] T. Munakata, Y. Jani, Fuzzy systems: an overview, Communications of the ACM 37 (3) (1994) 68-76.

[12] M. Sugeno, I. Hirano, S. Nakamura, S. Kotsu, Development of an intelligent unmanned helicopter, in: Fuzzy Systems, 1995. International Joint Conference of the Fourth IEEE International Conference on Fuzzy Systems and The Second International Fuzzy Engineering Symposium., Proceedings of 1995 IEEE Int, Vol. 5, IEEE, 1995, pp. 33-34.

[13] R.-E. Precup, H. Hellendoorn, A survey on industrial applications of fuzzy control, Computers in Industry 62 (3) (2011) 213-226.

[14] J. C. Doyle, K. Glover, P. P. Khargonekar, B. A. Francis, State-space solutions to standard h 2 and h control problems, Automatic Control, IEEE Transactions on 34 (8) (1989) 831-847.

[15] R. Babuska, Fuzzy modeling for control, Kluwer Academic Publishers, 1998.

[16] J. Abonyi, R. Babuska, F. Szeifert, Modified Gath-Geva fuzzy clustering for identification of Takagi-Sugeno fuzzy models, Systems, Man and Cybernetics, Part B, IEEE Transactions on 32 (5) (2002) 612-621.

[17] T. Takagi, M. Sugeno, Fuzzy identification of systems and its applications to modelling and control, IEEE Trans. on Systems, Man and Cybernetics 15(1) (1985) 116-132.

[18] C. W. Scherer, Lpv control and full block multipliers, Automatica 37 (3) (2001) 361-375.

[19] J. Shamma, M. Athans, Gain scheduling: potential hazards and possible remedies, IEEE Control Systems 12 (3) (1992) 101-107. doi:10.1109/37.165527.

[20] K. Tanaka, M. Sugeno, Stability Analysis of Fuzzy Systems Using Lyapunov's Direct Method, in: Proc. North American Fuzzy Information Society Annual Meeting (NAFIPS'90), Toronto, 1990, pp. 133-136.

[21] K. Tanaka, M. Sugeno, Stability analysis and design of fuzzy control systems, Fuzzy sets and systems 45 (2) (1992) 135-156.

[22] K. Tanaka, H. O. Wang, Fuzzy control systems design and analysis, Ed. John Wiley \& Sons, New York, USA, 2001.

[23] B. Kosko, Fuzzy systems as universal approximators, Computers, IEEE Transactions on 43 (11) (1994) $1329-1333$.

[24] J. L. Castro, M. Delgado, Fuzzy systems with defuzzification are universal approximators, Systems, Man, and Cybernetics, Part B: Cybernetics, IEEE Transactions on 26 (1) (1996) 149-152.

[25] T. Hastie, R. Tibshirani, J. Friedman, The elements of statistical learning, Springer, 2009.

[26] S. Skogestad, I. Postlethwaite, Multivariable feedback control: analysis and design, Wiley New York, 1996.

[27] H. D. Tuan, P. Apkarian, T. Narikiyo, M. Kanota, New fuzzy control model and dynamic output feedback parallel distributed compensation, Fuzzy Systems, IEEE Transactions on 12 (1) (2004) 13-21.

[28] J.-J. E. Slotine, W. Li, et al., Applied nonlinear control, Prentice-Hall, Englewood Cliffs, NJ, 1991.

[29] K. S. Ray, D. D. Majumder, Application of circle criteria for stability analysis of linear siso and mimo systems associated with fuzzy logic controller, Systems, Man and Cybernetics, IEEE Transactions on 14 (2) (1984) 345-349.

[30] K. S. Ray, A. M. Ghosh, D. D. Majumder, L 2-stability and the related design concept for siso linear system associated with fuzzy logic controller, Systems, Man and Cybernetics, IEEE Transactions on 14 (6) (1984) 932-939. 
[31] A. Ollero, J. Aracil, A. Garcia-Cerezo, Robust design of rule-based fuzzy controllers, Fuzzy Sets and Systems 70 (2) (1995) 249-273.

[32] F. Cuesta, F. Gordillo, J. Aracil, A. Ollero, Stability analysis of nonlinear multivariable takagi-sugeno fuzzy control systems, Fuzzy Systems, IEEE Transactions on 7 (5) (1999) 508-520.

[33] S. Boyd, L. El Ghaoui, E. Feron, V. Balakrishnan, Linear matrix inequalities in system and control theory, Ed. SIAM, Philadelphia, USA, 1994.

[34] H. O. Wang, K. Tanaka, M. Griffin, An analytical framework of fuzzy modeling and control of nonlinear systems: stability and design issues, in: American Control Conference, Proceedings of the 1995, Vol. 3, IEEE, 1995, pp. 2272-2276.

[35] H. O. Wang, K. Tanaka, M. F. Griffin, An approach to fuzzy control of nonlinear systems: stability and design issues, Fuzzy Systems, IEEE Transactions on 4 (1) (1996) 14-23.

[36] J. F. Sturm, Using SeDuMi 1.02, a Matlab toolbox for optimization over symmetric cones, Optimization Methods and Software 11 (1-4) (1999) 625-653.

URL http: / / www.tandfonline.com/doi/abs/10.1080/10556789908805766

[37] M. Johansson, Piecewise quadratic stability of fuzzy systems, IEEE Trans. Fuzzy Syst. 7 (1999) $713-722$.

[38] H. Lam, F. Leung, P. Tam, Stable and robust fuzzy controller for uncertain nonlinear systems based on a multiple-grid-point approach, in: Industrial Electronics, Control and Instrumentation, 1997. IECON 97. 23rd International Conference on, Vol. 1, IEEE, 1997, pp. 354-359.

[39] T.-M. Guerra, W. Perruquetti, Non-quadratic stabilisation of discrete takagi sugeno fuzzy models, in: Fuzzy Systems, 2001. The 10th IEEE International Conference on, Vol. 3, IEEE, 2001, pp. 1271-1274.

[40] K. Tanaka, T. Hori, H. Wang, A multiple Lyapunov function approach to stabilization of fuzzy control systems, Fuzzy Systems, IEEE Transactions on 11 (4) (2003) 582-589.

[41] T. Guerra, L. Vermeiren, LMI-based relaxed nonquadratic stabilization conditions for nonlinear systems in the takagi-sugeno's form, Automatica 10 (2004) 823 - 829.

[42] A. Kruszewski, T. Guerra, New approaches for the stabilization of discrete takagi-sugeno fuzzy models, Proc. IEEE CDC/ECC, Seville, Spain.

[43] H. Tuan, P. Apkarian, T. Narikiyo, Y. Yamamoto, Parameterized linear matrix inequality techniques in fuzzy control system design, IEEE Trans. Fuzzy Systems 9 (2) (2001) 324-332.

[44] X. Liu, Q. Zhang, New approaches to $H_{\infty}$ controller designs based on fuzzy observers for T-S fuzzy systems via LMI, Automatica 39 (9) (2003) 1571-1582.

[45] Y.-Y. Cao, P. M. Frank, Analysis and synthesis of nonlinear time-delay systems via fuzzy control approach, Fuzzy Systems, IEEE Transactions on 8 (2) (2000) 200-211.

[46] K. R. Lee, J. H. Kim, F. T. Jeung, H. B. Park, Output feedback robust h control of uncertain fuzzy dynamic systems with time-varying delay, Fuzzy Systems, IEEE Transactions on 8 (6) (2000) 657-664.

[47] X.-P. Guan, C.-L. Chen, Delay-dependent guaranteed cost control for TS fuzzy systems with time delays, Fuzzy Systems, IEEE Transactions on 12 (2) (2004) 236-249.

[48] B. Chen, X. Liu, Delay-dependent robust control for t-s fuzzy systems with time delay, Fuzzy Systems, IEEE Transactions on 13 (4) (2005) 544-556.

[49] E. T. Jeung, H. B. Park, et al., Delay-dependent control for time-delayed ts fuzzy systems using descriptor representation, International Journal of Control, Automation, and Systems 2 (2) (2004) 182-188.

[50] L.-X. Wang, Adaptive fuzzy systems and control: design and stability analysis, Prentice-Hall, Inc., Englewood Cliffs, NJ, 1994.

[51] R. Ordónez, K. M. Passino, Stable multi-input multi-output adaptive fuzzy/neural control, Fuzzy Systems, IEEE Transactions on 7 (3) (1999) 345-353. 
[52] L.-X. Wang, Stable adaptive fuzzy control of nonlinear systems, Fuzzy Systems, IEEE Transactions on 1 (2) (1993) 146-155.

[53] H.-X. Li, S. Tong, A hybrid adaptive fuzzy control for a class of nonlinear mimo systems, Fuzzy Systems, IEEE Transactions on 11 (1) (2003) 24-34.

[54] J. A. Farrell, M. M. Polycarpou, Adaptive approximation based control: Unifying neural, fuzzy and traditional adaptive approximation approaches, Vol. 48, John Wiley \& Sons, 2006.

[55] S. Labiod, M. S. Boucherit, T. M. Guerra, Adaptive fuzzy control of a class of mimo nonlinear systems, Fuzzy sets and systems 151 (1) (2005) 59-77.

[56] S. Tong, B. Chen, Y. Wang, Fuzzy adaptive output feedback control for mimo nonlinear systems, Fuzzy Sets and Systems 156 (2) (2005) 285-299.

[57] A. Boulkroune, M. Tadjine, M. MSaad, M. Farza, How to design a fuzzy adaptive controller based on observers for uncertain affine nonlinear systems, Fuzzy Sets and Systems 159 (8) (2008) 926-948.

[58] B. Ding, H. Sun, P. Yang, Further studies on lmi-based relaxed stabilization conditions for nonlinear systems in takagi-sugeno's form, Automatica 42 (3) (2006) 503-508.

[59] B. Ding, Homogeneous polynomially nonquadratic stabilization of discrete-time takagi-sugeno systems via nonparallel distributed compensation law, Fuzzy Systems, IEEE Transactions on 18 (5) (2010) 994-1000.

[60] D. H. Lee, J. B. Park, Y. H. Joo, Approaches to extended non-quadratic stability and stabilization conditions for discrete-time takagi-sugeno fuzzy systems, Automatica 47 (3) (2011) 534-538.

[61] A. Megretski, Integral quadratic constraints derived from the set-theoretic analysis of difference inclusions, in: Decision and Control, 1996., Proceedings of the 35th IEEE Conference on, Vol. 3, 1996, pp. 2389-2394 vol.3. doi:10.1109/CDC.1996.573446.

[62] A. Kruszewski, T. Guerra, New Approaches for the Stabilization of Discrete Takagi-Sugeno Fuzzy Models, 44th IEEE Decision and Control and 2005 European Control Conf. (2005) 3255-3260.

[63] A. Kruszewski, R. Wang, T.-M. Guerra, Nonquadratic stabilization conditions for a class of uncertain nonlinear discrete time ts fuzzy models: a new approach, Automatic Control, IEEE Transactions on 53 (2) (2008) 606-611.

[64] T.-M. Guerra, H. Kerkeni, J. Lauber, L. Vermeiren, An efficient lyapunov function for discrete t-s models: observer design, Fuzzy Systems, IEEE Transactions on 20 (1) (2012) 187-192.

[65] Z. Lendek, T.-M. Guerra, J. Lauber, Controller design for TS models using delayed nonquadratic Lyapunov functions, IEEE Trans. on Cybernetics In press (2015) doi:10.1109/TCYB.2014.2327657.

[66] L. A. Mozelli, R. M. Palhares, F. Souza, E. M. Mendes, Reducing conservativeness in recent stability conditions of ts fuzzy systems, Automatica 45 (6) (2009) 1580-1583.

[67] J.-T. Pan, T. M. Guerra, S.-M. Fei, A. Jaadari, Nonquadratic stabilization of continuous t-s fuzzy models: Lmi solution for a local approach, Fuzzy Systems, IEEE Transactions on 20 (3) (2012) 594-602.

[68] F. Wu, K. Dong, Gain-scheduling control of $\{$ LFT $\}$ systems using parameter-dependent lyapunov functions, Automatica 42 (1) (2006) 39 - 50. doi : http: / / dx. doi.org/10.1016/j.automatica. 2005. 08.020 .

URL http: //www.sciencedirect.com/science/article/pii/s0005109805003213

[69] B.-J. Rhee, S. Won, A new fuzzy lyapunov function approach for a takagi-sugeno fuzzy control system design, Fuzzy sets and systems 157 (9) (2006) 1211-1228.

[70] L. A. Mozelli, R. M. Palhares, G. S. Avellar, A systematic approach to improve multiple lyapunov function stability and stabilization conditions for fuzzy systems, Information Sciences 179 (8) (2009) 1149-1162.

[71] T.-M. Guerra, M. Bernal, A way to escape from the quadratic framework, in: Fuzzy Systems, 2009. FUZZIEEE 2009. IEEE International Conference on, IEEE, 2009, pp. 784-789.

[72] M. Bernal, T. M. Guerra, Generalized nonquadratic stability of continuous-time takagi-sugeno models, Fuzzy Systems, IEEE Transactions on 18 (4) (2010) 815-822. 
[73] D. H. Lee, D. W. Kim, Relaxed Imi conditions for local stability and local stabilization of continuoustime Takagi-Sugeno fuzzy systems, Cybernetics, IEEE Transactions on 44 (3) (2014) 394-405. doi: $10.1109 /$ TCYB.2013.2256781.

[74] E. Tian, C. Peng, Delay-dependent stability analysis and synthesis of uncertain t-s fuzzy systems with timevarying delay, Fuzzy sets and systems 157 (4) (2006) 544-559.

[75] O. Kwon, M.-J. Park, S.-M. Lee, J. H. Park, Augmented lyapunov-krasovskii functional approaches to robust stability criteria for uncertain takagi-sugeno fuzzy systems with time-varying delays, Fuzzy Sets and Systems 201 (2012) 1-19.

[76] C. Peng, M.-R. Fei, An improved result on the stability of uncertain t-s fuzzy systems with interval timevarying delay, Fuzzy Sets and Systems 212 (2013) 97-109.

[77] H.-B. Zeng, J. H. Park, J.-W. Xia, S.-P. Xiao, Improved delay-dependent stability criteria for t-s fuzzy systems with time-varying delay, Applied Mathematics and Computation 235 (2014) 492-501.

[78] F. O. Souza, V. C. Campos, R. M. Palhares, On delay-dependent stability conditions for Takagi-Sugeno fuzzy systems, Journal of the Franklin Institute 351 (7) (2014) 3707 - 3718. doi : http: / / dx. doi. org/10.1016/j.jfranklin.2013.03.017.

[79] L. Zhao, H. Gao, H. R. Karimi, Robust stability and stabilization of uncertain t-s fuzzy systems with timevarying delay: an input-output approach, Fuzzy Systems, IEEE Transactions on 21 (5) (2013) 883-897.

[80] B. Chen, X. Liu, C. Lin, K. Liu, Robust h control of takagi-sugeno fuzzy systems with state and input time delays, Fuzzy Sets and Systems 160 (4) (2009) 403-422.

[81] X. Song, S. Xu, H. Shen, Robust h control for uncertain fuzzy systems with distributed delays via output feedback controllers, Information Sciences 178 (22) (2008) 4341-4356.

[82] B. Øksendal, Stochastic differential equations, Springer, 2003.

[83] Z. Wang, D. W. Ho, X. Liu, A note on the robust stability of uncertain stochastic fuzzy systems with timedelays, Systems, Man and Cybernetics, Part A: Systems and Humans, IEEE Transactions on 34 (4) (2004) 570-576.

[84] B. Zhang, S. Xu, G. Zong, Y. Zou, Delay-dependent stabilization for stochastic fuzzy systems with time delays, Fuzzy Sets and Systems 158 (20) (2007) 2238-2250.

[85] T. Senthilkumar, P. Balasubramaniam, Delay-dependent robust h control for uncertain stochastic t-s fuzzy systems with time-varying state and input delays, International Journal of Systems Science 42 (5) (2011) $877-887$.

[86] S. Prajna, A. Papachristodoulou, F. Wu, Nonlinear control synthesis by sum of squares optimization: a Lyapunov-based approach, Control Conference, 2004. 5th Asian 1.

[87] G. Chesi, On the gap between positive polynomials and sos of polynomials, Automatic Control, IEEE Transactions on 52 (6) (2007) 1066-1072.

[88] K. Tanaka, H. Yoshida, H. Ohtake, H. O. Wang, Stabilization of polynomial fuzzy systems via a sum of squares approach, in: Intelligent Control, 2007. ISIC 2007. IEEE 22nd International Symposium on, IEEE, 2007, pp. 160-165.

[89] K. Tanaka, H. Yoshida, H. Ohtake, H. O. Wang, A sum of squares approach to stability analysis of polynomial fuzzy systems, in: American Control Conference, 2007. ACC'07, IEEE, 2007, pp. 4071-4076.

[90] A. Sala, Reducing the gap between fuzzy and nonlinear control, in: Proc. 3rd IFAC Conf. on Adv. Fuzzy Neural Control, 2007, pp. 121-126.

[91] A. Sala, C. Arino, Polynomial fuzzy models for nonlinear control: a taylor series approach, Fuzzy Systems, IEEE Transactions on 17 (6) (2009) 1284-1295.

[92] K. Tanaka, H. Ohtake, H. O. Wang, Guaranteed cost control of polynomial fuzzy systems via a sum of squares approach, Systems, Man, and Cybernetics, Part B: Cybernetics, IEEE Transactions on 39 (2) (2009) 561-567. 
[93] A. Sala, On the conservativeness of fuzzy and fuzzy-polynomial control of nonlinear systems, Annual Reviews in Control 33 (1) (2009) 48-58.

[94] K. Tanaka, H. Ohtake, T. Seo, M. Tanaka, H. O. Wang, Polynomial fuzzy observer designs: a sum-ofsquares approach, Systems, Man, and Cybernetics, Part B: Cybernetics, IEEE Transactions on 42 (5) (2012) $1330-1342$.

[95] M. Bernal, A. Sala, A. Jaadari, T.-M. Guerra, Stability analysis of polynomial fuzzy models via polynomial fuzzy lyapunov functions, Fuzzy Sets and Systems 185 (1) (2011) 5-14.

[96] Y.-J. Chen, M. Tanaka, K. Tanaka, H. Ohtake, H. O. Wang, Brief paper-discrete polynomial fuzzy systems control, Control Theory \& Applications, IET 8 (4) (2014) 288-296.

[97] J. L. Pitarch, A. Sala, C. V. Arino, Closed-form estimates of the domain of attraction for nonlinear systems via fuzzy-polynomial models, IEEE Tran. on Cybernetics 44 (4) (2014) 526-538.

[98] H. Li, Z. Chen, Y. Sun, H. R. Karimi, Stabilization for a class of nonlinear networked control systems via polynomial fuzzy model approach, Complexity (2014) In Pressdoi : 10.1002/ cplx.21579.

[99] H.-K. Lam, F. H.-F. Leung, Stability Analysis of Fuzzy-model-based Control Systems: Linear-matrixinequality Approach, Vol. 264, Springer, 2011.

[100] A. Sala, C. Arino, Relaxed stability and performance lmi conditions for takagi-sugeno fuzzy systems with polynomial constraints on membership function shapes, Fuzzy Systems, IEEE Transactions on 16 (5) (2008) 1328-1336.

[101] A. Sala, T. M. Guerra, Stability analysis of fuzzy systems: membership-shape and polynomial approaches, in: Proc. IFAC World Congress, 2008, pp. 5605-5610.

[102] A. Sala, Introducing shape-dependent relaxed conditions in fuzzy control of nonlinear systems in takagisugeno form, in: Fuzzy Systems, 2008. FUZZ-IEEE 2008.(IEEE World Congress on Computational Intelligence). IEEE International Conference on, IEEE, 2008, pp. 512-517.

[103] C. Ariño, A. Sala, Relaxed LMI conditions for closed loop fuzzy systems with tensor product structure, Eng. Appl. Artif. Intell. 20 (8) (2007) 1036-1046.

[104] M. Bernal, T.-M. Guerra, A. Jaadari, Non-quadratic stabilization of takagi-sugeno models: a local point of view, in: Fuzzy Systems (FUZZ), 2010 IEEE International Conference on, IEEE, 2010, pp. 1-6.

[105] A. Sala, C. Ariño, Assymptotically necessary and sufficient conditions for stability and performance in fuzzy control: Applications of Polya's theorem, Fuzzy Sets and Systems 158 (4) (2007) 2671-2686.

[106] A. Kruszewski, A. Sala, T.-M. Guerra, C. Ariño, A triangulation approach to asymptotically exact conditions for fuzzy summations, Fuzzy Systems, IEEE Transactions on 17 (5) (2009) 985-994.

[107] C. Scherer, LMI Relaxations in Robust Control, European Journal of Control 1 (2006) 3-29, european Journal of Control.

[108] K. Tanaka, H. Yoshida, H. Ohtake, H. Wang, Stabilization of Polynomial Fuzzy Systems via a Sum of Squares Approach, Intelligent Control, 2007. ISIC 2007. IEEE 22nd International Symposium on (2007) 160-165.

[109] T. Hu, F. Blanchini, Non-conservative matrix inequality conditions for stability/stabilizability of linear differential inclusions, Automatica 46 (1) (2010) 190-196.

[110] C. Ariño, E. Pérez, A. Sala, F. Bedate, Polytopic invariant and contractive sets for closed-loop discrete fuzzy systems, Journal of the Franklin Institute 351 (7) (2014) 3559-3576.

[111] C. V. Ariño, A. Sala, Shape-dependent maximal controllable sets for constrained discrete-time TS systems, in: Proc. IEEE Int. Conf. on Fuzzy Systems (FUZZ-IEEE), IEEE Press, 2015, p. In Press.

[112] Y. Yang, C. Zhou, Adaptive fuzzy h stabilization for strict-feedback canonical nonlinear systems via backstepping and small-gain approach, Fuzzy Systems, IEEE Transactions on 13 (1) (2005) 104-114.

[113] M. Wang, B. Chen, S.-L. Dai, Direct adaptive fuzzy tracking control for a class of perturbed strict-feedback nonlinear systems, Fuzzy sets and systems 158 (24) (2007) 2655-2670. 
[114] Y.-J. Liu, S.-C. Tong, W. Wang, Adaptive fuzzy output tracking control for a class of uncertain nonlinear systems, Fuzzy Sets and Systems 160 (19) (2009) 2727-2754.

[115] Y.-J. Liu, S.-C. Tong, T.-S. Li, Observer-based adaptive fuzzy tracking control for a class of uncertain nonlinear mimo systems, Fuzzy Sets and Systems 164 (1) (2011) 25-44.

[116] A. Boulkroune, M. MSaad, M. Farza, Adaptive fuzzy controller for multivariable nonlinear state timevarying delay systems subject to input nonlinearities, Fuzzy Sets and Systems 164 (1) (2011) 45-65.

[117] S. Tong, Y. Li, Adaptive fuzzy output feedback tracking backstepping control of strict-feedback nonlinear systems with unknown dead zones, Fuzzy Systems, IEEE Transactions on 20 (1) (2012) 168-180.

[118] Y. Li, S. Tong, T. Li, Adaptive fuzzy output-feedback control for output constrained nonlinear systems in the presence of input saturation, Fuzzy Sets and Systems 248 (2014) 138-155.

[119] S. J. Qin, T. A. Badgwell, A survey of industrial model predictive control technology, Control engineering practice 11 (7) (2003) 733-764.

[120] I. Atacak, O. F. Bay, A type-2 fuzzy logic controller design for buck and boost dc-dc converters, Journal of Intelligent Manufacturing 23 (4) (2012) 1023-1034.

[121] H. Lam, H. Li, C. Deters, E. Secco, H. Wurdemann, K. Althoefer, Control design for interval type-2 fuzzy systems under imperfect premise matching, Industrial Electronics, IEEE Transactions on 61 (2) (2014) 956968. doi:10.1109/TIE.2013.2253064.

[122] C. Arino, A. Sala, Extensions to stability analysis of fuzzy control systems subject to uncertain grades of membership, Systems, Man, and Cybernetics, Part B: Cybernetics, IEEE Transactions on 38 (2) (2008) 558-563. doi:10.1109/TSMCB.2007.913596. 